\title{
Kink dynamics in a novel discrete sine-Gordon system
}

\author{
J M Speight* and R S Ward \\ Department of Mathematical Sciences, \\ University of Durham, Durham DH1 3LE
}

\begin{abstract}
A spatially-discrete sine-Gordon system with some novel features is described. There is a topological or Bogomol'nyi lower bound on the energy of a kink, and an explicit static kink which saturates this bound. There is no Peierls potential barrier, and consequently the motion of a kink is simpler, especially at low speeds. At higher speeds, it radiates and slows down.
\end{abstract}

AMS Classification numbers 58F, 70F.

\section{Introduction}

There are many nonlinear systems, in one spatial dimension, which admit topologically-stable kink solutions: for example, the sine-Gordon and phi-four systems. They have many physical applications. For applications in, say, condensed-matter physics or biophysics, an accurate model should take the discreteness of space into account - in other words, the kinks live on a one-dimensional lattice rather than in a continuum. A discrete version of the sine-Gordon system which has been extensively studied is the Frenkel-Kontorova model, in which the partial derivative $\partial / \partial x$ of the continuum system is replaced by the forward difference on the lattice. In this case, much of the topological character of the model is lost, and there is no known explicit kink solution.

Let us briefly recall two of the features of kink motion in the Frenkel-Kontorova model [1-5]. First, the energy of a kink depends on its position in relation to the lattice: a static kink located halfway between two lattice sites has a lower energy than one located exactly on a lattice site. The difference between these two energies is the so-called Peierls-Nabarro barrier. So if a moving kink does not have enough kinetic energy, then it gets trapped between two adjacent lattice sites; it oscillates, and emits radiation, and cannot escape. Secondly, a kink which starts off with enough speed to surmount the Peierls-Nabarro barrier, and move along the lattice, will lose energy (as radiation) and slow down, until eventually it too becomes trapped.

In this note, we wish to describe an alternative discrete sine-Gordon system, in which the PeierlsNabarro barrier is eliminated. It maintains an important feature of the continuum model, namely a topological lower bound ("Bogomol'nyi bound") on the energy of a kink. And it admits an explicit static kink solution on the lattice, which saturates this lower bound. A moving kink will still radiate and slow down, but it is never trapped, no matter how slowly it moves. So this lattice sine-Gordon model is quite different from the usual one described above.

In the next section we shall describe the model. Subsequent sections study kink motion, for low speed and for (moderately) high speed. In each case, we make approximate analytic predictions of the kink behaviour, and compare these with numerical simulations.

${ }^{*}$ Current e-mail address: speight@amsta.leeds.ac.uk 


\section{A topological discrete sine-Gordon system}

Let us begin with a very brief review of the situation for the continuous sine-Gordon equation $\varphi_{x x}-\varphi_{t t}=\sin \varphi$. It is convenient to use the dependent variable $\psi=\frac{1}{2} \varphi$ instead of $\varphi$. The potential energy of the field is

$$
E_{P}=\frac{1}{4} \int_{-\infty}^{\infty}\left(\psi_{x}^{2}+\sin ^{2} \psi\right) d x
$$

(this is normalized so that a single static kink has unit energy). The kink boundary condition is $\psi \rightarrow 0$ as $x \rightarrow-\infty, \psi \rightarrow \pi$ as $x \rightarrow \infty$. The standard Bogomol'nyi argument [6] is

$$
\begin{aligned}
0 & \leq \frac{1}{4} \int_{-\infty}^{\infty}\left(\psi_{x}-\sin \psi\right)^{2} d x \\
& =E_{P}+\frac{1}{2} \int_{-\infty}^{\infty} \partial_{x}(\cos \psi) d x \\
& =E_{P}-1,
\end{aligned}
$$

where kink boundary conditions are imposed. So the energy $E_{P}$ is bounded below by 1 ; and $E_{P}$ equals 1 if and only if $\psi_{x}=\sin \psi$, the solution of which is the static kink

$$
\psi(x)=2 \arctan \exp \left(x-x_{0}\right) .
$$

From this point on, $x$ becomes a discrete variable, with lattice spacing $h$. The subscript + denotes forward shift, ie. $f_{+}(x)=f(x+h)$; so, for example, the forward difference is given by $\Delta f=h^{-1}\left(f_{+}-f\right)$. To obtain a lattice version of the Bogomol'nyi bound, we may begin with the same function $\cos \psi$ as appears in (2), and "reconstruct" the inequality. The first step is to choose a factorization

$$
\Delta \cos \psi=-D F
$$

where $D \rightarrow \psi_{x}$ and $F \rightarrow \sin \psi$ in the continuum limit $h \rightarrow 0$. Then define the potential energy of the lattice sine-Gordon field to be

$$
E_{P}=\frac{h}{4} \sum_{x=-\infty}^{\infty}\left(D^{2}+F^{2}\right)
$$

It follows, just as in the continuum case, that (with kink boundary conditions) $E_{P}$ is bounded below by 1 ; and the minimum is attained if and only if $D=F$.

There is a choice involved in (4), and we are free to make whatever choice we like. The most natural one seems to be

$$
\begin{aligned}
D & =\frac{2}{h} \sin \frac{1}{2}\left(\psi_{+}-\psi\right), \\
F & =\sin \frac{1}{2}\left(\psi_{+}+\psi\right)
\end{aligned}
$$

Substituting these into (5) gives

$$
E_{P}=\frac{h}{4} \sum_{x=-\infty}^{\infty}\left[\frac{4}{h^{2}} \sin ^{2} \frac{1}{2}\left(\psi_{+}-\psi\right)+\sin ^{2} \frac{1}{2}\left(\psi_{+}+\psi\right)\right]
$$

which, of course, reduces to (1) in the continuum limit.

Let us summarize what we have so far. The real-valued field $\psi(t, x)$ depends on the continuous variable $t$ and the discrete variable $x$. Its potential energy $E_{P}$ is defined by the expression (7); for the kinetic energy, we may for example make the simple choice

$$
E_{K}=\frac{h}{4} \sum_{x=-\infty}^{\infty} \dot{\psi}^{2}
$$


where $\dot{\psi}=d \psi / d t$. The boundary condition on $\psi$ is that it should tend to an integer multiple of $\pi$ (independent of time) as $x \rightarrow-\infty$ or $x \rightarrow+\infty$; this guarantees finite energy. A 1-kink configuration has the boundary values $\psi \rightarrow 0$ as $x \rightarrow-\infty$ and $\psi \rightarrow \pi$ as $x \rightarrow+\infty$. For such fields, the total energy $E=E_{P}+E_{K}$ is bounded below by 1 ; and this lower bound is attained if and only if $\dot{\psi}=0$, and the two expressions in (6) are equal.

This latter condition, namely $D=F$, is called the Bogomol'nyi equation [6]. It is a first-order difference equation, whose solutions (for kink boundary conditions) minimize the potential energy. Hence these (static) solutions are also solutions of the Euler-Lagrange equations

$$
\ddot{\psi}=-\frac{2}{h} \frac{\partial E_{P}}{\partial \psi}
$$

since $\partial E_{P} / \partial \psi=0$ at a minimum. This is a general feature of the Bogomol'nyi argument: one gets first-order equations whose solutions are also static solutions of the second-order equations of motion. Furthermore (and this is the most important feature), solutions of the Bogomol'nyi equations have an energy which is at its topological minimum value.

In the expression (7) for $E_{P}$, the first term in the summand represents an attractive force (nonlinear Hooke's law) which couples nearest neighbours, and the second term a substrate potential depending on the average of pairs of nearest neighbours. The strengths of these two forces have, in effect, been normalized by scaling, and $h$ may be regarded as a dimensionless parameter in the model.

The Bogomol'nyi equation $D=F$ may also be written as

$$
\tan \frac{\psi_{+}}{2}=\frac{2+h}{2-h} \tan \frac{\psi}{2}
$$

from which one sees that the parameter $h$ should be less than 2 if one is to obtain a well-behaved solution. In fact, the solution of (10) can be written down explicitly: given that $0<h<2$, it is

$$
\psi=2 \arctan \exp a(x-b),
$$

where

$$
a=\frac{2}{h} \operatorname{arctanh} \frac{h}{2}
$$

and where $b$ is an arbitrary real constant. This is a static lattice kink solution. Because it corresponds to a minimum of the energy in the kink sector, it is stable under perturbations which remain in that sector; in other words, under perturbations which preserve the kink boundary conditions on $\psi$. The location of the kink is determined by the continuous parameter (zero-mode) $b$. The kink is highly localized, in the sense that its energy density (the summand in eqn 7 ) is concentrated on just a couple of links of the lattice. For example, if $b=0$, then the proportion of the energy contributed by the two links $[-h, 0]$ and $[0, h]$ is $4 h /\left(4+h^{2}\right)$; if $h=1$ this proportion is $80 \%$, and as $h \rightarrow 2$ it tends to $100 \%$. A diagram illustrating the kink solution $\psi(x)$ for $h=1$ is presented in figure 1 .

It is instructive to look at the limit $h=2$. In this case, the solution of the Bogomol'nyi equation is supported at a single lattice site $x_{0}$ :

$$
\psi(x)= \begin{cases}0 & \text { if } x<x_{0} \\ c & \text { if } x=x_{0} \\ \pi & \text { if } x>x_{0}\end{cases}
$$

where $c$ is a real constant. By contrast with (11), the "position" zero-mode is discrete; and there is an additional continuous zero-mode, namely the value $c$ of $\psi$ at the centre of the kink. This limiting case is therefore marginally unstable: a perturbation will in general cause $\psi\left(x_{0}\right)$ to increase or decrease without bound. 


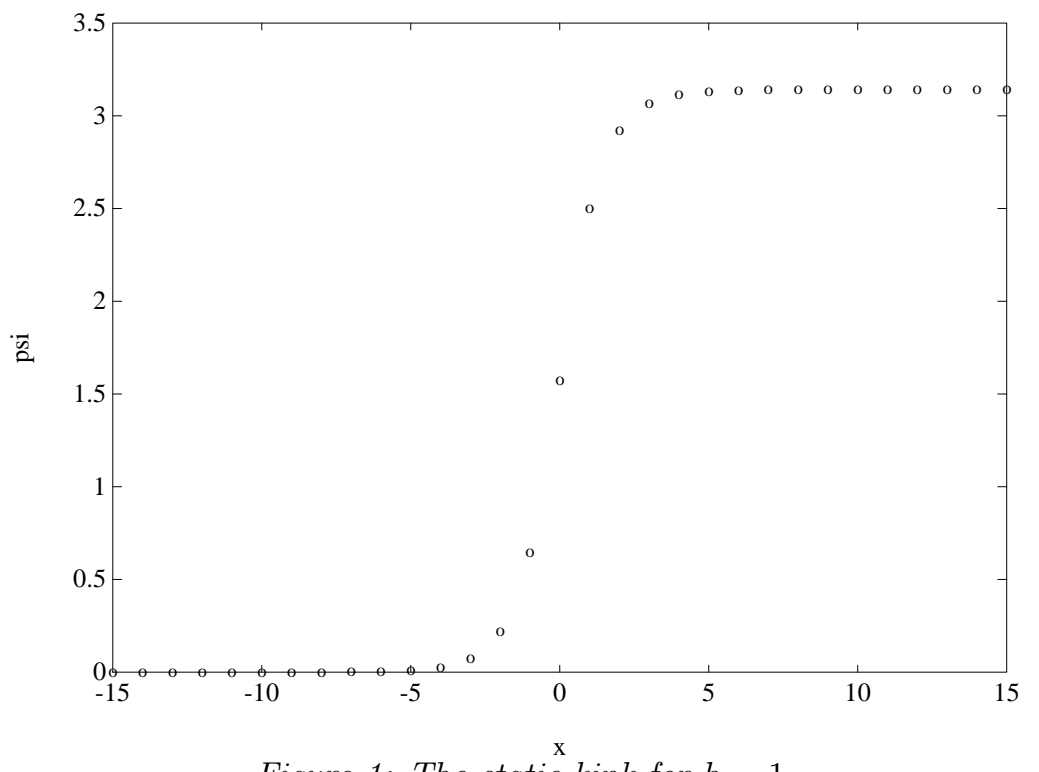

Figure 1: The static kink for $h=1$.

The situation we wish to study is that of the moving kink. There is no explicit solution in this case, and so one has to resort to approximation, or to numerical solution of the equations of motion (9), namely

$$
\begin{aligned}
\ddot{\psi} & =\frac{1}{h^{2}}\left[\sin \left(\psi_{+}-\psi\right)-\sin \left(\psi-\psi_{-}\right)\right]-\frac{1}{4}\left[\sin \left(\psi_{+}+\psi\right)+\sin \left(\psi+\psi_{-}\right)\right] \\
& =\frac{4-h^{2}}{4 h^{2}} \cos \psi\left(\sin \psi_{+}+\sin \psi_{-}\right)-\frac{4+h^{2}}{4 h^{2}} \sin \psi\left(\cos \psi_{+}+\cos \psi_{-}\right)
\end{aligned}
$$

\section{A collective-coordinate approximation}

The simplest approximation of kink dynamics is obtained by restricting $\psi$ to have the form (11), with $b$ now becoming a dynamical variable $b(t)$. So the number of degrees of freedom is reduced from infinity to one. Our conjecture is that this appproximation is a good one, provided that the speed $v$ of the kink is small ( $\mathrm{cf}[$, 8 ) ; we shall elaborate on this condition later in the section. So the Lagrangian is

$$
\begin{aligned}
L & =E_{K}-E_{P} \\
& =f(b) \dot{b}^{2}-1,
\end{aligned}
$$

where

$$
f(b)=\frac{a^{2} h}{4} \sum_{x=-\infty}^{\infty} \operatorname{sech}^{2} a(x-b) .
$$

Note that $f(b)$ is periodic with period $h$, is even, and has a local maximum at $b=0$. Graphs of $f(b)$ for various values of $h$ are given in figure 2 .

The Euler-Lagrange equation of the system is

$$
f(b) \ddot{b}=-f^{\prime}(b) \dot{b}^{2} .
$$

This may be reduced to quadratures:

$$
v t=\int_{0}^{b(t)} \sqrt{\frac{f(\tilde{b})}{f(0)}} d \tilde{b}
$$




$$
\equiv F_{h}(b)
$$

where $b(0)=0, \dot{b}(0)=v$. The function $F_{h}(b)$ is strictly increasing, and satisfies

$$
F_{h}(b+n h)=F_{h}(b)+n F_{h}(h)
$$

for all integers $n$ owing to the periodicity of $f(b)$, so it suffices to know $F_{h}(b)$ for $0 \leq b<h$.

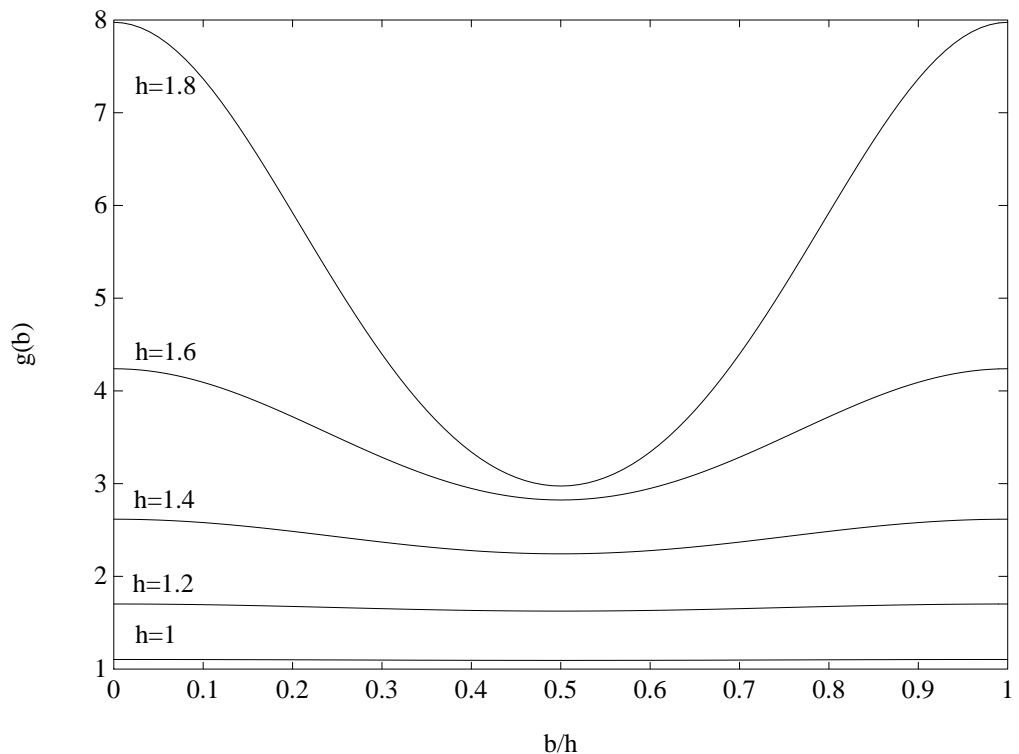

Figure 2: The function $f(b)$ of equation (10).

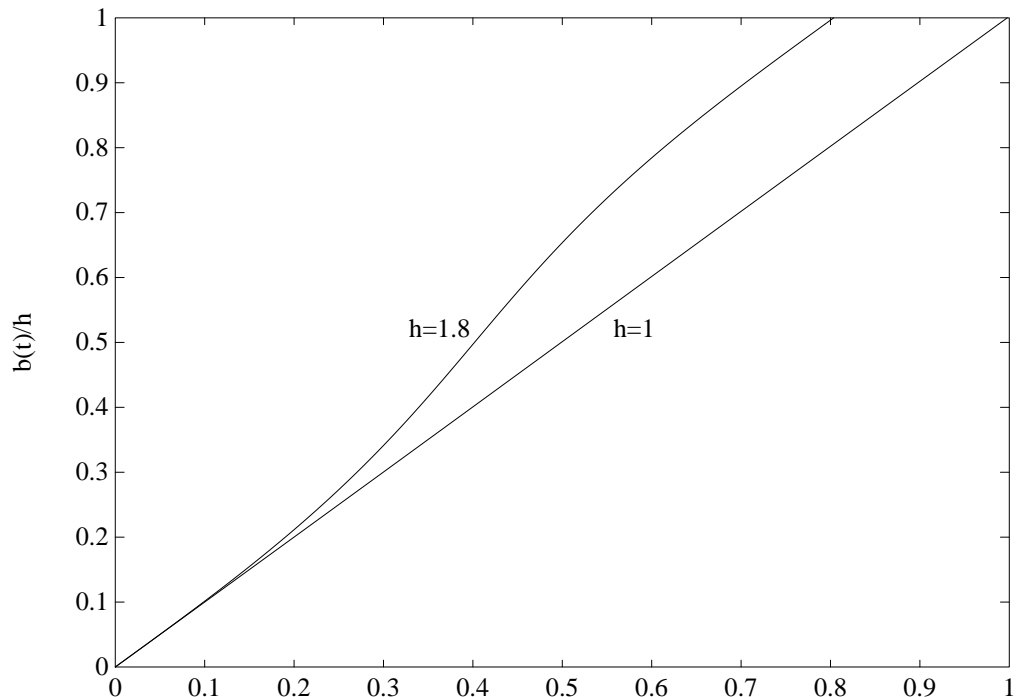

Figure 3: The function $b(t)$ over one wobble period for $h=1$ and $h=1.8$.

This function $F_{h}$ is easily inverted (fig. 3 ) to give the kink trajectory

$$
b(t)=F_{h}^{-1}(v t) .
$$

The main feature is that the kink wobbles as it moves through the lattice. This wobble is a dynamical effect; there is of course no static potential (such as Peierls-Nabarro) causing it. The time taken for 
the kink to move from $x=0$ to $x=h$ (the wobble period) is

$$
T=\frac{F_{h}(h)}{v} ;
$$

and $F_{h}(h) / h<1$, approaching the upper bound in the continuum limit, $h \rightarrow 0$. That is, the kink travels faster by a factor $h / F_{h}(h)$ than would be naïvely suggested by the initial velocity $v$. A graph of $F_{h}(h)$ (fig. 4) shows that discreteness effects are small for $h<1.2$, but grow large as $h$ approaches 2.

We believe that this approximation of kink motion is accurate for small $v$. The Bogomol'nyi bound is crucial to this belief: the idea is that the configuration sits at the bottom of a onedimensional potential valley, and the kink can move freely along the valley floor (ie. in the direction of the zero-mode $b$ ). There are at least two ways in which the approximation differs from the full model. First, there is dynamical dressing (a moving kink has a different shape from a static one). In the continuum limit, this correction is of order $v^{2}$ (relativistic contraction), and one might expect the dressing in the discrete case also to be of order $v^{2}$ (bearing in mind that the expression for the static kink is exact). Secondly, there is transfer of energy to radiation (phonons). In Bogomol'nyi field theories, the suggestion is that this energy transfer will be suppressed by a factor of order $\exp \left(-T_{0} / T_{1}\right)$, where $T_{0}$ is a typical timescale for the truncated dynamics, and $T_{1}$ is the maximum period of the radiation in the theory [8]. Our suggestion (not proved) is that the same is true in Bogomol'nyi lattice models such as the present one. In this case, $T_{0}$ is the wobble period (21), which is of order $h / v$; and $T_{1}=2 \pi$, since the minimum phonon frequency is unity (see next section). So the energy transfer (per lattice site traverse) is suppressed by $\exp [-h /(2 \pi v)]$, and is therefore negligible for small $v$ (as long as $h$ is not small). If $h$ is small, in fact if $h$ is less than 1, then the amplitude of the wobble is tiny, and so one would expect the radiation to be negligible irrespective of $v$; of course, this is exactly what happens in the continuum $(h \rightarrow 0)$ limit.

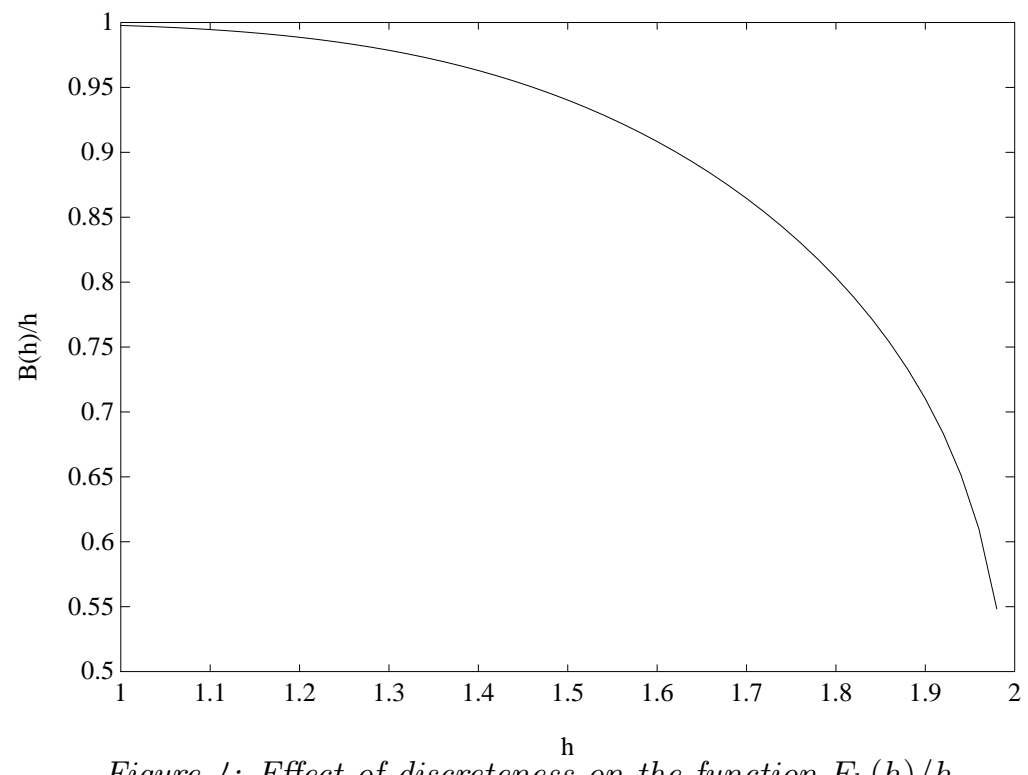

Figure 4: Effect of discreteness on the function $F_{h}(h) / h$.

The accuracy of the approximation has been tested numerically using a fully-explicit fourth-order Runge-Kutta algorithm with fixed time-step 0.01. The initial condition was a Galilean-boosted static kink profile with initial velocity 0.01 lattice sites per unit time $(v=0.01 h)$. Simulations of duration 1000 time units were performed for $h=1,1.2,1.4,1.6$ and 1.8. In every case the kink moves freely, without pinning, undergoing motion of the predicted periodicity. Furthermore, inspection of the kink velocity over a single period reveals close agreement with $\dot{b}(t)$ calculated from (20) (see fig. 5), 
although for $h=1.2$, numerical errors rather swamp the very small theoretical wobble (note the scale on the velocity axis).

Collective-variable analyses of the Frenkel-Kontorova model (cf [5]) have introduced the kink position as an extra variable, accompanied by a constraint. But since the dynamics is much more complicated, all the degrees of freedom have to be kept in order to obtain accurate results. A truncation to one degree of freedom, as in the present case, does not work.
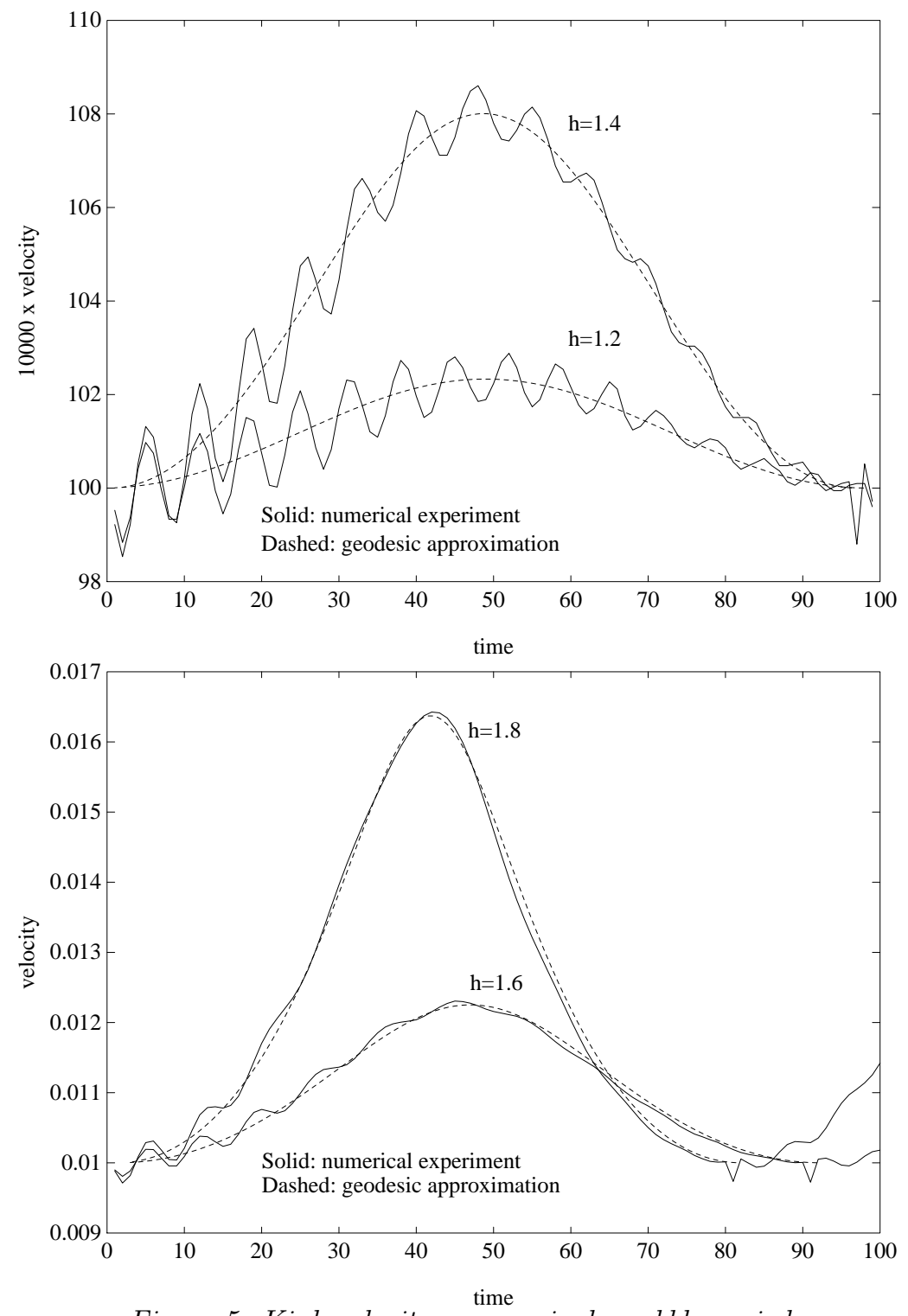

Figure 5: Kink velocity over a single wobble period.

\section{Fast-moving kinks}

The collective-coordinate approximation is expected to fail at high velocities (except for small $h$ ). This is observed in the simulations as a gradual onset of kink deceleration as the initial velocity is increased. Failure occurs at lower velocities for coarser lattices-around $v=0.012$ for $h=1.8$ compared with $v=0.15$ for $h=1$. The kink energy is dissipated in the form of small amplitude 
oscillations ("radiation" or "phonons") emitted in its wake, propagating backwards (see fig. 6).

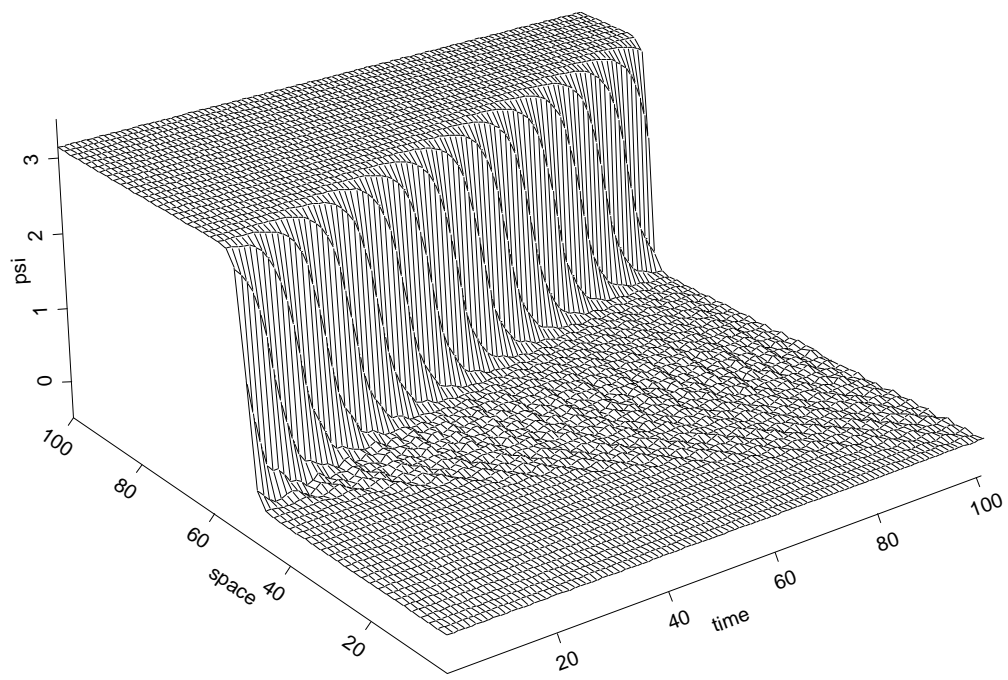

Figure 6: Radiation by a fast-moving kink.

The effect of this radiation on the kink velocity over a long time-scale can be seen in fig. 7 . The data were produced by the above-mentioned Runge-Kutta algorithm, run for 16000 time units with a time-step of 0.01 . The initial configuration was a static kink Galilean-boosted to speed $v=0.3$, on a lattice of unit spacing $(h=1)$. To cut reflexion of radiation from the fixed left-hand boundary, the first five lattice sites were damped. After an initial velocity drop of 0.02 in 10 time units as the kink assumes a more appropriate shape, it decelerates more slowly. The modulation of the amplitude of velocity oscillations is due to the velocity sampling (once every 10 time units) falling in and out of phase with the periodic wobble of the kink as it passes lattice sites.

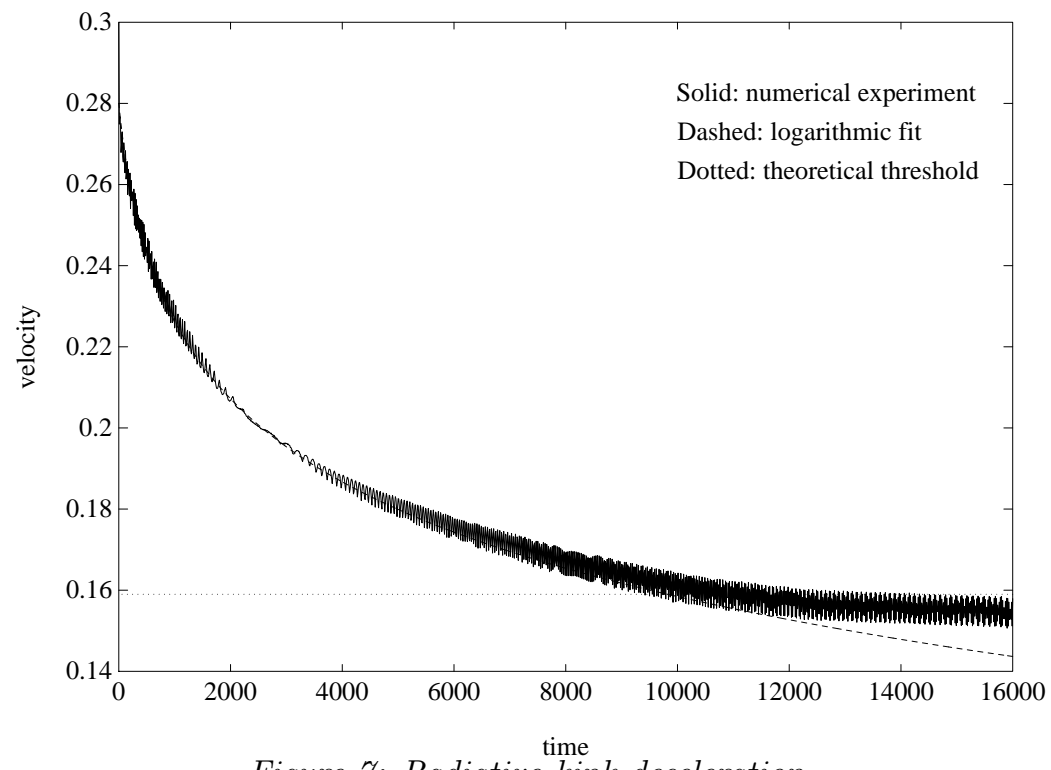

Figure 7: Radiative kink deceleration. 
The most interesting feature of fig. 7 is the existence of a threshold velocity, $v \approx 0.16$, below which deceleration, and hence radiation, is much reduced. Some understanding of this phenomenon may be gained by an analysis (motivated by [4]) of the linearized equations of motion,

$$
\ddot{\psi}=\frac{4-h^{2}}{4 h^{2}}\left(\psi_{+}+\psi_{-}\right)-\frac{4+h^{2}}{2 h^{2}} \psi .
$$

From this one derives a dispersion relation for small-amplitude travelling waves, namely,

$$
\omega^{2}=\frac{4+h^{2}}{2 h^{2}}-\frac{4-h^{2}}{2 h^{2}} \cos k h
$$

where $k$ is the wave-number. The angular frequency $\omega$ ranges between 1 and $2 / h$, the lower bound being responsible for the threshold velocity.

As the kink travels along the lattice, it hits lattice sites with frequency $v / h$ sites per unit time. Provided $v \geq h / 2 \pi$, the kink can excite radiation of the same frequency, that is $\omega=2 \pi v / h$. However, if $v<h / 2 \pi$, then $\omega<1$, and the lattice cannot support such radiation. The kink can only excite higher harmonics, so the rate of energy dissipation is suddenly cut and the kink velocity becomes "quasi-stable." For $h=1$, this threshold occurs at $v=1 / 2 \pi \approx 0.159$, in good agreement with the numerical data (fig. 7).

A theoretical understanding of the specific shape of the graph is more elusive. We make the ad-hoc assumption (motivated by figure 7 of [ 顿) that the energy $\Delta E$ lost by a kink in traversing a single lattice cell at speed $v$ obeys an exponential law:

$$
\Delta E=e^{p v-q},
$$

where $p$ and $q$ are positive constants, properties of the lattice. We further assume that the kinetic energy of the kink is

$$
E_{K}=\frac{1}{2} c v^{2}
$$

where $c$ is approximately constant provided $h$ is not too large, as suggested by the results of section 3 (fig. 2). These two equations imply a first order differential equation for $v(t)$, easily solved to give

$$
v(t)=v(0)-A \log (B t+1),
$$

where

$$
\begin{aligned}
A & =\frac{1}{p}, \\
B & =\frac{p}{h c} e^{p v(0)-q} .
\end{aligned}
$$

The dashed curve in fig. 7 is a fit of this formula to the numerical data, taking $A=0.032, B=$ $0.004365, v(0)=0.28$. Estimating $c=1.0986$ by averaging the function $f(b)$ for $h=1$, one deduces that $p=31.3$, and $q=17.5$. The fit is good for velocities greater than the radiation threshold at $v=0.159$.

\section{Conclusion}

We have described a spatially-discrete sine-Gordon system, which is significantly different from the usual (Frenkel-Kontorova) system. There is a "topological" lower bound on the kink energy, and an explicit static kink solution with this energy. The kink moves on a "level playing-field": there is no Peierls-Nabarro potential barrier. In particular, the behaviour of a kink moving with low speed is much simpler. 
The idea described in section 2 for generating "topological" discrete systems, can be applied to many other continuum theories which have a Bogomol'nyi bound on the energy of a kink. By way of example, consider the $\phi^{4}$ equation, where the continuum potential energy density is

$$
\frac{1}{4}\left(\frac{\partial \varphi}{\partial x}\right)^{2}+\frac{1}{4}\left(1-\varphi^{2}\right)^{2}
$$

In equation (4), one replaces $\cos \psi$ by $\frac{1}{3} \varphi^{3}-\varphi$. The most obvious factorization is then

$$
\begin{aligned}
D & =\Delta \varphi \\
F & =1-\frac{1}{3}\left(\varphi_{+}^{2}+\varphi_{+} \varphi+\varphi^{2}\right) .
\end{aligned}
$$

The lattice potential energy is then given by (5) as before, and the kink energy is bounded below by $2 / 3$ (in the kink sector $\varphi \rightarrow \pm 1$ as $x \rightarrow \pm \infty$ ).

It would be interesting to investigate breathers and kink-antikink collisions in this sine-Gordon model. Again, the absence of the Peierls potential should lead to behaviour which differs qualitatively from the usual model [9]. One simple observation is that in the limiting case $h=2$, there exists a breather located at a single lattice site. Indeed, the field

$$
\psi(t, x)= \begin{cases}0 & \text { if } x \neq 0 \\ \theta(t) & \text { if } x=0\end{cases}
$$

satisfies the equation of motion if and only if $\theta(t)$ satisfies the pendulum equation

$$
\ddot{\theta}=-\sin \theta \text {. }
$$

Acknowledgement: JMS is supported by a research studentship awarded by the UK Science and Engineering Research Council.

\section{References}

[1] Currie J F, Trullinger S E, Bishop A R and Krumhansl J A, 1977 Numerical simulation of sine-Gordon soliton dynamics in the presence of perturbations Phys. Rev. B 15 5567-5580.

[2] Ishimori Y and Munakata T, 1982 Kink dynamics in the discrete sine-Gordon system-A perturbational approach J. Phys. Soc. Jpn. 51 3367-3374.

[3] Combs J A and Yip S, 1983 Single-kink dynamics in a one-dimensional atomic chain: A nonlinear atomistic theory and numerical simulation Phys. Rev. B 28 6873-6885.

[4] Peyrard M and Kruskal M D, 1984 Kink dynamics in the highly discrete sine-Gordon system Physica 14D 88-102.

[5] Boesch R, Willis C R and El-Batanouny M, 1989 Spontaneous emission of radiation from a discrete sine-Gordon kink Phys. Rev. B 40 2284-2296.

[6] Bogomol'nyi E B, 1976 The stability of classical solutions Sov. J. Nucl. Phys. 24 449-454.

[7] Manton N S, 1982 A remark on the scattering of BPS monopoles Phys. Lett. 110B 54-56.

[8] Manton N S, 1982 Unstable manifolds and soliton dynamics Phys. Rev. Lett. 60 1916-1919.

[9] Boesch R and Peyrard M, 1991 Discreteness effects on a sine-Gordon breather Phys. Rev. B 43 8491-8508. 